\title{
Analysis of the GRID's Basic Topological Structure Reliability
}

\author{
Vladimir E. Gvozdev, Lilia R. Chernyakhovskaya, \\ Darya V. Blinova, Dinara R. Akhmetova
}

Department of Computer Science and Robotics, Ufa State Aviation Technical University, 12 K. Marks St, 450007 Ufa, Russia

e-mail: gvozdev.ve@net.ugatu.su, chernyahovskaya.lr@net.ugatu.su, blinova.dv@net.ugatu.su, ahmetova.dinara-r@net.ugatu.su

\begin{abstract}
Approaches to the investigation of the reliability of the basic topological structures of GRID systems, which are minimal enough set of constructive elements for constructing such systems, are considered in this work. Methods used for the quantitative assessment of the reliability of basic topological structures under conditions of different interpretations of the concept of "full failure of the basic structure" are considered. The construction of structural reliability models, based on series-parallel reliability schemes, are described in this work. The application of these approaches creates the basis for a multi-dimensional analysis of the GRID-systems reliability characteristics, at the design and development stages. This, in turn, creates the prerequisites for assessing the functional safety of such systems.
\end{abstract}

Keywords: GRID; topology; reliability; failure

\section{Introduction}

The tendency to improve the efficiency of computing and storage resources has served as the basis for the emergence of a focus in the field of information technologies, especially, GRID systems.

In the work [1], with references to other literature [2, 3], three key indicators of the effectiveness of GRID-systems are listed, including reliability. The problem of GRID systems reliability management is a priority for world-wide research efforts. There exists the need of development of methodological, methodical bases and tools of reliability management, at different stages system's components life cycle.

The main feature of GRID-systems is the possibility of redistribution of computing resources and resources, for data storage, it follows that the reliability 
analysis of such systems depends on the reliability of information processing nodes and the reliability of communication facilities [4-6].

Reliability is the characteristic of the systems, retaining in time, within the established limits and values for all parameters, that characterize the ability to perform the required functions in the specified modes and conditions of use.

In the works [1, 7], basic topologies are distinguished, called by the author, the basic fractal architectures as follows:

- Complete cellular topology

- $\quad$ Ring topology

- Topology "star"

- Linear topology

- $\quad$ Mixed topology

Allocated components of GRID systems play the role of typical aggregates, that consist of two types of elements: data processing (storage) unit and equipment that provides communication of nodes. In the mentioned work it is emphasized, that the selected aggregates are the basic constructions from which GRID-systems of any complexity can be built. Due to this, the task of GRID-systems aggregates reliability, can be singled out as an independent task. In the work [1], an approach to assessing the reliability of basic topological structures (BTS) is described. This approach based on the analysis of graphs connectivity, where nodes are computers, workstations, concentrators, and edges are communication channels.

In this paper approaches to quantifying the reliability of basic topological structures are considered. In the present work, an approach to quantitative assessment of the basic topological structures, sensitivity indicators to the change in the reliability of nodes and connections under various definitions of the concept of "failure of BTS GRID systems" on the basis of the well-known apparatus of series-parallel logic circuits [8]. Building the reliability models of BTS is the basis for assessing the reliability of GRID systems at the design and development stage, which, creates prerequisites for assessing the functional safety of such systems.

\section{Characterization of GRID Systems Components Reliability Studies}

Analysis of published sources allows for identification of the following areas of research, related to managing the reliability of the components of GRID-systems: 
- Methods for software testing (components, interfaces, stress, security, system, etc.), verification and validation. A significant number of publications, manuals and standards are devoted to this theme $[9,10,11]$

- Development of theoretical and methodical bases of the analysis of reliability and safety of info-communication systems of various scale and complexity $[1,12-16]$

- Development of theoretical basis and tools for designing of error-steady systems [17-21]

- Development of theoretical foundations, manuals and decision support tools at the design stage according to the reliability criteria [10, 22-24]

- Development of methodological, methodical and model bases of a new scientific direction of software defectology $[15,25]$

Analysis of the references allows to make a conclusion, that the task of managing reliability of GRID-systems belongs to the class of tasks of managing complex systems.

\section{The Approaches to Assess the Reliability of Basic Topological Structures of GRID-Systems}

The basis of the analysis of the reliability of complex technical systems, an integral component of which is software, is the concept of "failure." In the wellknown literature [1] it is shown that the concept of "failure" is treated differently depending on the functional architecture of the systems [10], the point of view of the target users group [26], the system's conditions of functioning [27]. The interpretation of the concept of "failure of a part of the GRID system" by stakeholders and target groups of users is based on their subjective idea of the desired consumer properties of the systems [25].

As an independent problem of reliability of technical systems, the problem of reliability of the elements making up this system is singled out in the classical theory of reliability. In the framework of this study, the basic topological structures are considered as a minimal sufficient set of aggregates from which a GRID system can be built. Taking into account that GRID-systems are related to complex systems, it is possible to adapt the provisions of the reliability theory for the reliability analysis of GRID.

In the theory of reliability, approaches to the study of repaired and non-repaired systems are distinguished. In the construction of the models presented below, postulated the independence of failures of nodes (computers, workstations, routers, communication devices) and edges (communication channels) and the 
provision for unrepairable components. This assumption is valid, firstly, when the emphasis in research is on the hardware component of the BTS, and secondly, when the current repair of a technical object is technically impossible at the site of its operation or is economically inexpedient. In the framework of this paper, it is assumed that the element can be either in the working or in the inoperative state, the situation of partial loss of efficiency is not considered.

The approach to estimate the reliability of non-repaired systems using serialparallel logic schemes is known. Below are given examples of structural-logical schemes for a linear basic architecture, corresponding to the case when it consists of four nodes.

\section{Evaluation of Reliability of the Basic Topological Structure "Linear" depending on the Content of the "System Failure" Concept}

\subsection{Evaluation of the Basic Topological Structure Reliability by the Criterion of the Isolation of the Components of the Graph}

Described in this section models are based on the following interpretation of the concept of "full failure": a complete disruption of the GRID network (all nodes of this network are isolated) due to non-repairable failure of nodes (computers, workstations, routers, communication devices) or edges. Taking into account the main feature of GRID-systems, this designation is formulated as follows: There was a full failure of the system, if there is no any possibility of reallocation of resources.

In accordance with this interpretation of the concept of failure, a serial-parallel scheme is constructed to evaluate the reliability of a typical aggregate of the basic architecture "linear" consisting of 4 nodes (Figs. 1-2). In this case, the communication channel between all the elements is a bus.

This paper considers the characteristic of BTS reliability as a time between failures and also assumes that all blocks on the above structural serial-parallel scheme have the same value of probability $p$.

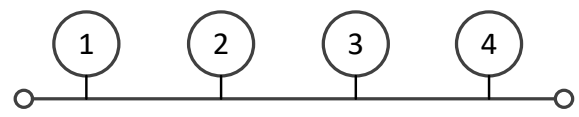

Figure 1

Logical structure "linear" 


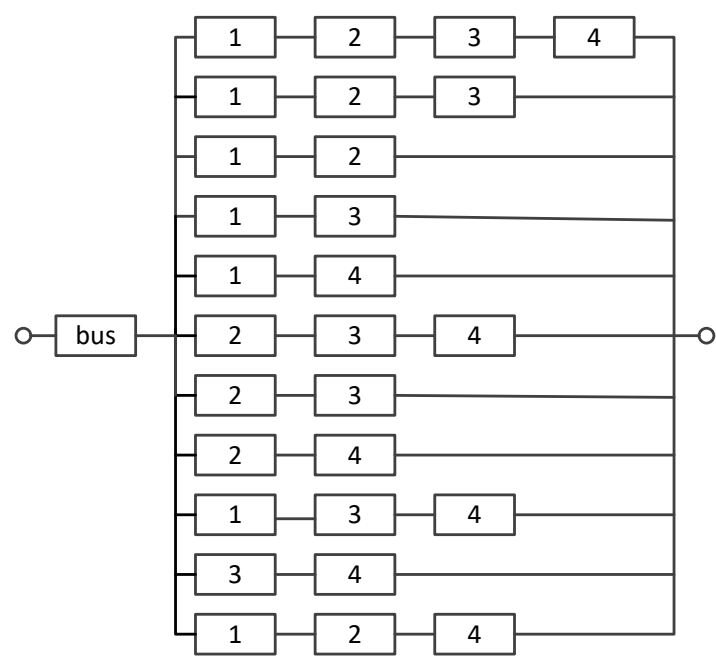

Figure 2

Serial-parallel scheme of reliability (all nodes are isolated)

Proceeding from the aforementioned, for the basic topological structure "linear" consisting of four nodes, the following relation can be put in correspondence for calculating the reliability of the system:

$$
p_{\text {BTS }}=p_{\text {bus }} *\left(1-\left(1-p^{4}\right) *\left(1-p^{3}\right)^{4} *\left(1-p^{2}\right)^{6}\right)
$$

Analogous dependencies were obtained for the topological structure "linear" consisting of five and six nodes, the plots of the dependence of $p_{B T S}$ on the value of $p$ are shown in Fig. 3.

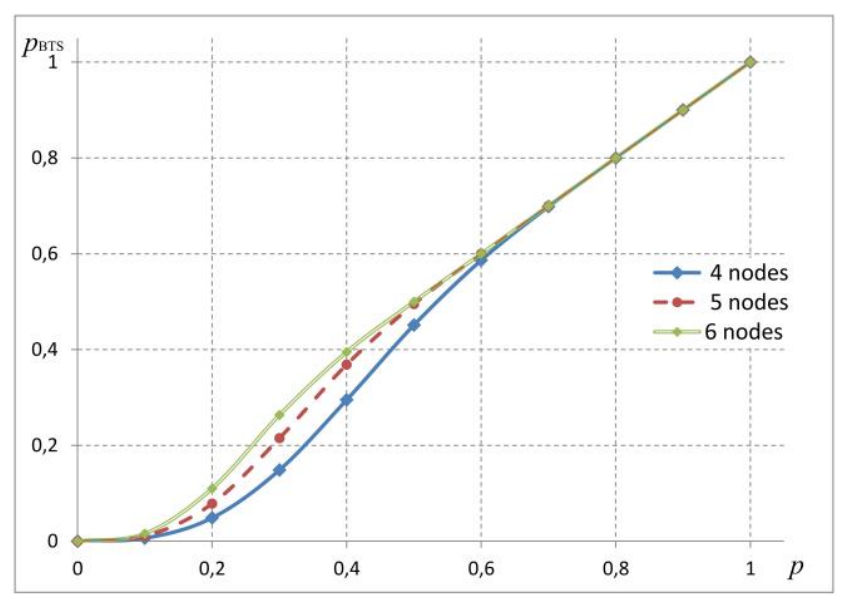

Figure 3

Dependence the system's reliability on the elements reliability (all nodes are isolated) 
From the dependencies shown in Figure 3 it follows that within the framework of the introduced "full failure" concept, the reliability of the component increases with the increase in the number of nodes.

\subsection{Evaluation of the Basic Topological Structure Reliability by the Criterion of the Absence of a Connection between Any Two Components of the Graph}

In this section, the concept of "total failure" is interpreted as: violation of the GRID network workability (there is no connection between any two nodes) nonrepairable failure of nodes (computers, workstations, routers, communication devices) or edges (communication channels). This interpretation of the concept of failure is given in [1]. A serial-parallel logic scheme on the basis of which the reliability of a linear BTS is evaluated will look like it is shown in Fig. 4. Taking into account the main feature of GRID-systems, this definition is formulated as follows: there was a full failure of the system, if there was a failure of any element, which leads to the impossibility of redistributing resources between all elements.

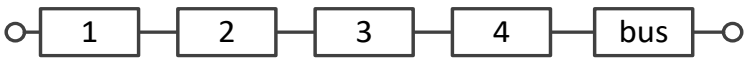

Figure 4

Serial-parallel scheme of reliability (there is no connection between any two nodes)

For the considered serial-parallel logical scheme of the BTA "linear", the following relationship for calculating the reliability of the system can be used:

$$
p_{B T S}=p^{5}
$$

The dependencies of $p_{B T S}$ on the value of $p$ for "linear" BTA with four, five and six nodes are given on Fig. 5.

From the dependencies shown in Figure 5 it follows that within the framework of the introduced "full failure" concept, the reliability of the component decreases with the increase in the number of nodes.

As can be seen from the given examples, even in the framework of one basic topology, the results of the construction of serial-sequential schemes differ depending on the interpretation of the concept of "full failure". This, in turn, leads to obtaining of various calculation formulas for assessing the reliability of the BTS and the various dependencies of reliability of the elements on the reliability of the unit.

Building the reliability assessments is a complex task, at the center of which is a failure, the concept of which is treated differently by each target group of users with their valuable concepts. 


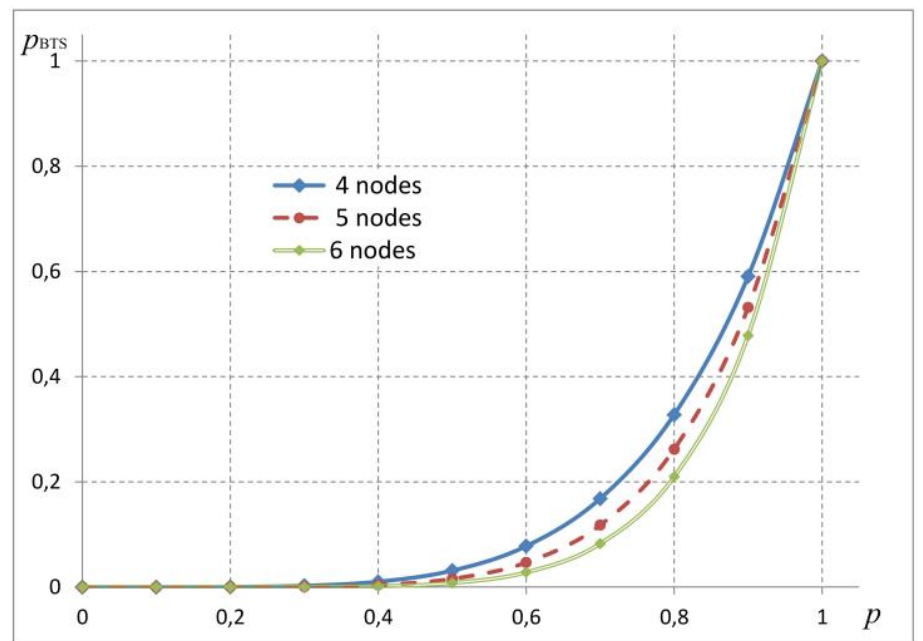

Figure 5

Dependence the system's reliability on the elements reliability (there is no connection between any two nodes)

\section{Analysis of the Reliability Sensitivity of a Basic Topological Structure "Linear" to the Reliability of Its Elements}

The sensitivity characteristic allows to make an informed conclusion about how the increase in the reliability of the elements is affects the change in the reliability of the structure as a whole.

Figs. 6-7 show the sensitivity of the reliability characteristics of the system $p_{B T S}$ from the reliability characteristics values of architecture elements, obtained on the basis of the ratio:

$$
S=\frac{\Delta p_{B T S}}{\Delta p}
$$

As can be seen from the figures, the formulation of the concept of "failure", affects a change in the reliability characteristics of the unit, depending on the reliability characteristics of its elements. 


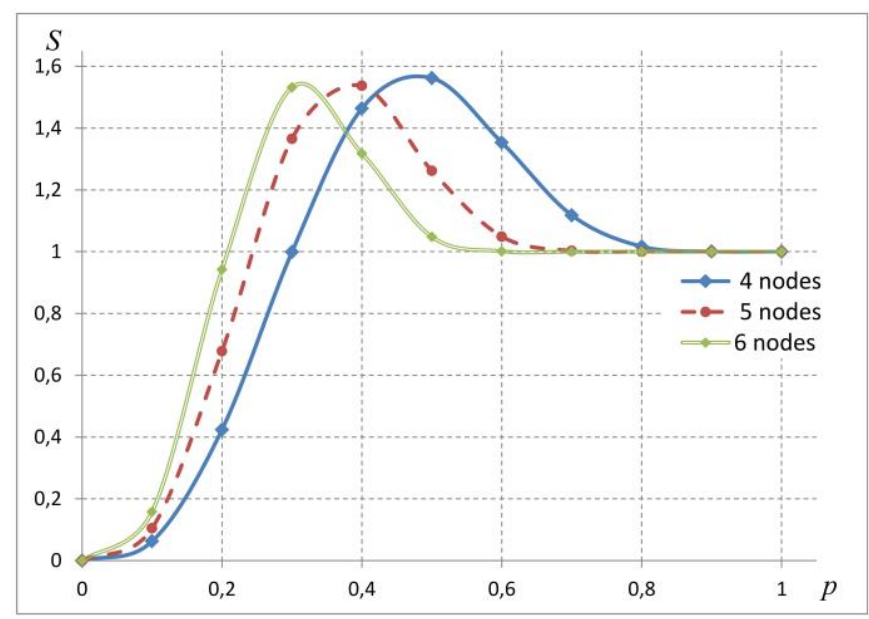

Figure 6

Sensitivity of system reliability to reliability of elements (all nodes are isolated)

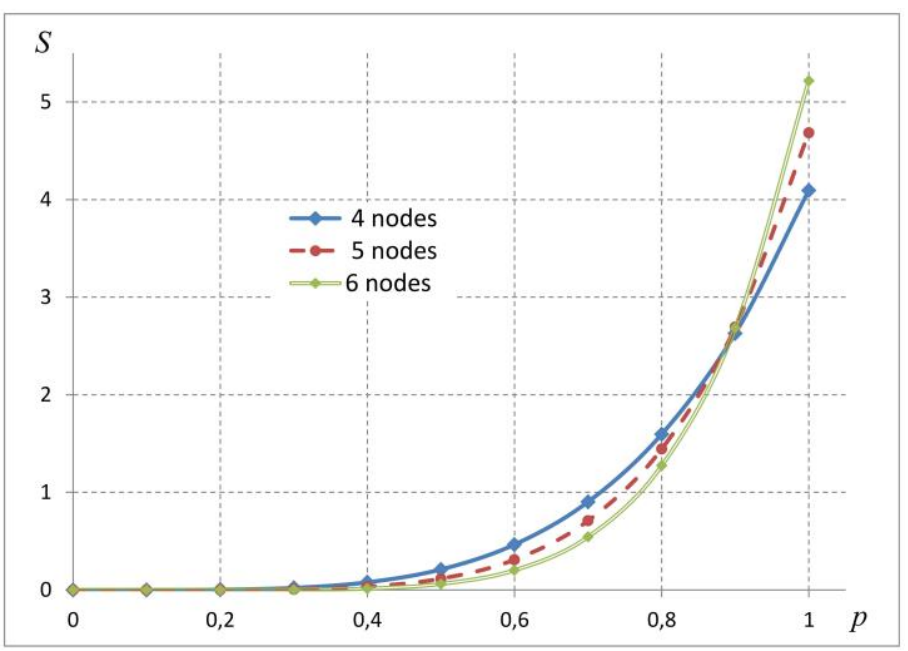

Figure 7

Sensitivity of system reliability to reliability of elements

(there is no connection between any two nodes)

\section{Conclusion}

The selection of basic topological structures makes it possible to represent any distributed GRID systems in the form of a set of standard aggregates. In this regard, as a scientific problem with applied value, it is necessary to single out the task of analyzing the reliability of the basic topological structures that make up the basis for the analysis of design solutions in the construction of GRID systems. 
The GRID reliability analysis is a multi-criteria task and a system approach should be the methodological basis for its solution.

GRID systems are used to meet the information needs of different users with completely different system requirements, that affects the interpretation of the concept "failure".

Due to the fact that the basic units are hardware-software complexes, different contents of the concept "failure" can be put in correspondence with them, which effects on the reliability characteristics of the basic topological structures and GRID-systems with different interpretation of the concept of reliability.

Depending on research objectives, it is possible to construct a set of models of failures (models of operational state) GRID. The choice of a model is determined by the specific features of the tasks within the framework of ensuring functional safety (survivability, storability, etc.).

As an example of a model that supplements the previously described models, we give the failure model of the linear topology GRID shown in Fig. 1 by the criterion "the possibility of interaction of any nodes of the system." Failure in this case will be understood as the absence of communication between any two nodes at any time due to non-recoverable failure of nodes (computers, workstations, routers, communication devices) or edges (communication channels, bus, terminators).

In this interpretation of the "failure" concept, such condition of GRID is possible, when all nodes are in working order, but are isolated from each other. In this case, it is impossible to reallocate resources, which is a failure of GRID. The failure model is shown in Fig. 8.

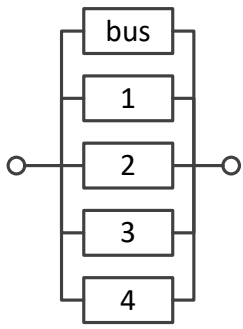

Figure 8

Serial-parallel scheme of failure (there is no connection between any two nodes)

As future research, the authors plan to develop a bank (matrix) of models for different formulations of reliability and failure, depending on the level of the GRID interaction protocols (physical, logical, applied).

\section{Acknowledgments}

This research was supported by grant 19-08-00177 of Russian Foundation for Basic Research. 


\section{References}

[1] Timofeev, A. V.: Adaptive control and intellectual analysis of information flows in computer networks, Anatoliya, Moscow, 2016

[2] Halabi, S., McPherson, D.: Internet routing architectures, second edition, Cisco Press, Indianapolis, 2001

[3] Walrand, J., Varaiya, P.: High-Performance Communication Networks, $2^{\text {nd }}$ Edition, Morgan Kaufmann, 1999

[4] Katic, N.: Performance analysis of smart grid solutions in distribution power systems, Acta Polytechnica Hungarica Vol. 15, No. 6, 2018, pp. 7997

[5] Thierion, V., Ayral, P., Jacob, G.: Grid technology reliability for flash flood forecasting: end-user assessment, Journal of Grid Computing, Vol. 9, 2011, pp. $405-422$

[6] Islam, T. Z., Bagchi, S., Eigenmann, R.: Reliable and efficient distributed checkpointing system for grid environments, Journal of Grid Computing, Vol. 12, 2014, pp. 593-613

[7] Timofeev, A. V., Dimitrichenko D. P.: Multi-criteria evaluation for network topological structures for GRID-systems modeling and design, Proc of SPIIRAN, 2009; Vol. 11, pp. 193-212

[8] Kapur, K., Lamberson, L.: Reliability in engineering design, Wiley India Pvt. Limited, 2009

[9] Myers G. J. "Software reliability: Principles and practices". Wiley, New York, 1976

[10] ESA PSS-05-10 Guide to software verification and validation, March 1995

[11] IEEE STD 1012 Approved Draft Standard for System, Software and Hardware Verification and Validation, 2016

[12] IEC 60300-3-1:2003 "Dependability management - Part 3-1: Application guide - Analysis techniques for dependability - Guide on methodology"

[13] IEC 61165:1995 Application of Markov techniques (MOD)

[14] Lee D. Y., Vouk M., Williams L. "Using Software Reliability Models for Security Assessment - Verification of Assumptions", In proc. of ISSRE 2013, pp. 23-24

[15] Borodakij, Yu. V., Yusupov, R. M., Palchun, B. P.: Problem of imitation modelling of computing infosphere defectoscopy features, Proc. of the $3^{\text {rd }}$ Russian conf. «Imitation modelling. Theory and Practice» Sankt-Peterburg, 2007, pp. 87-92

[16] Gvozdev, V. E., Blinova, D. V., Akhmetova, D. R.: Statistical analysis of time of establishing steady phases of functioning of complex hardware- 
software systems, 2018 International Russian Automation Conference (RusAutoCon), 2018, pp. 1-5

[17] Morozov, A., Janschek, K., Yusupova, N.: On the influence of control from properties to software error location, Proc. of the International Workshop "Innovation of Information Technologies - Theory and Practice", Dresden, Germany, 2010, pp. 86-91

[18] Michael, C. C., Jones, R. C.: On the uniformity of error propagation in software, Proc. of the Annual Conference on Computer Assurance (COMPASS'97), 1997, pp. 68-76

[19] Ammar, H., Nassar, D., AbdelMoez, W., Shereshevsky, M. "A framework for Experimental Error Propagation Analysis of Software Architecture Specifications”. In: FastAbstract ISSRE, 2002

[20] Aguilar, J. A., Garrigós, I., Mazón, J.-N.: Requirements Engineering in the Development Process of Web Systems: A Systematic Literature Review, Acta Polytechnica Hungarica Vol. 13, No. 3, 2016, pp. 61-80

[21] Nethravathi, B., Kamalesh, V. N.: Computation of maximal connectivity for fault tolerance: graph theoretical model and algorithm, International Journal of Grid and Distributed Computing, Vol. 13, No. 1, 2020, pp. 412417

[22] Pentti, H., Atte, H. "Failure mode and effect amalysis of software-based automatic systems". STVK-YTO-TR130, 2002

[23] Munson, J. C., Khoshgoflaar, T. M. "The detection of faultprone programs". IEEE Transactions on Software Engineering, 1992; 18(5): 423433

[24] Kan-Hin Chai, Jun Zhang, Kay-Chuan Tan: A TRIZ-Based Method for New Service Design". Journal of Service Research, National University of Singapore, 2005; 8(1): 48-66

[25] Gvozdev, V. E., Blinova, D. V., Chernyakhovskaya, L. R.: Prevention of defects on the early stages of designing hardware-software complexes on the basis of the positions of the theory of intersubjective management, Ontology of designing, 2016, 4(22), pp. 452-464

[26] Yussupova, N., Kovács, G., Boyko, M., Bogdanova, D.: Models and methods for quality management based on artificial intelligence applications, Acta Polytechnica Hungarica, Vol. 13, No. 3, 2016, pp. 45-60

[27] Gvozdev, V. E., Guzairov, M. B., Blinova, D. V., Davlieva, A. S.: Control of component alterations according with the target efficiency of data processing and control system, CEUR Workshop Proceedings, 2017, Vol. 1903, pp. 11-16 